\title{
Diagnosis and Treatment of Acute Kidney Injury in Pediatrics
}

Jennifer G. Jetton, $M D^{1}$

Erika T. Rhone, $M D^{2}$

Matthew W. Harer, $M D^{3}$

Jennifer R. Charlton, $M D, M S^{2}$

David T. Selewski, MD, MS ${ }^{4, *}$

\author{
Address \\ ${ }^{1}$ Division of Nephrology, Dialysis and Transplantation, Stead Family Department of \\ Pediatrics, University of Iowa Children's Hospital, Iowa City, IA, USA \\ ${ }^{2}$ Division of Nephrology, Department of Pediatrics, University of Virginia, \\ Charlottesville, VA, USA \\ ${ }^{3}$ Division of Neonatology, Department of Pediatrics, University of Virginia, \\ Charlottesville, VA, USA \\ ${ }^{*}, 4$ Division of Nephrology, Department of Pediatrics and Communicable Diseases, \\ C.S. Mott Children's Hospital, University of Michigan, Ann Arbor, MI, USA \\ Email: dselewsk@med.umich.edu
}

Published online: 16 March 2016

(C) Springer International Publishing AG 2016

This article is part of the Topical Collection on Pediatric Nephrology

Keywords Acute kidney injury $\cdot$ Pediatric $\cdot$ Continuous renal replacement therapy $\cdot$ Biomarker $\cdot$ Renal angina

\section{Opinion statement}

The term acute kidney injury (AKI) has replaced the outdated term acute renal failure throughout the literature and clinical practice. The term "injury" highlights the spectrum of organ injury that may occur and reflects the fact that even small changes in serum creatinine (rise of $0.3 \mathrm{mg} / \mathrm{dL}$ ) can be associated with adverse outcomes. A major advance in the field of AKI research has been the development of standardized staged definitions of AKI that allow for comparison of incidence, prevalence, and outcomes across studies. The Kidney Disease: Improving Global Outcomes (KDIGO) AKI definition represents the most recent consensus definition which is currently recommended for use in pediatric populations. Utilization of standard AKI definitions has made it clear that AKI occurs often in hospitalized patients and is associated with adverse short-term and long-term outcomes (hospital length of stay, mortality, subsequent chronic kidney disease). Awareness of the impact of AKI has resulted in increased efforts to understand, diagnose, prevent, and manage AKI earlier in the course of illness. While attempts at finding a treatment for AKI have been unsuccessful, largely due to the lack of sensitivity of the primary biomarker, serum creatinine, there have been many major advances in this field over the last 15 years. 
The development of novel biomarkers to predict the development of AKI in a timely manner and improve diagnostic accuracy is being pioneered by pediatric AKI researchers. The development of risk stratification scores (renal angina) and functional bedside tests (furosemide stress test) is enhancing our use of these biomarkers and our ability to predict those patients most likely to develop severe AKI. The recognition of the impact of fluid overload on mortality and hospital length of stay in patients with severe AKI has prompted more timely and frequent use of renal replacement therapy in critically ill children. Finally, we are recognizing that children who suffer AKI are at long-term risk for the development of chronic kidney disease and warrant follow-up.

\section{Introduction}

Acute kidney injury (AKI) is described as a sudden impairment in kidney function that results in decreased glomerular filtration rate, an inability to maintain fluid balance and electrolyte homeostasis, and an inability to handle waste products. There have been a number of major advances in the field of AKI research over the last 15 years thanks to growing recognition that even small changes in kidney function, previously thought to be of little consequence, can have a significant impact on short-term (hospital length of stay, mortality) and long-term outcomes (development of chronic kidney disease). Though a treatment for AKI remains elusive, much work has been done in the way we approach, diagnose, and manage AKI. The purpose of this review is to highlight the major advances in definition, epidemiology, outcomes, novel biomarker development, clinical awareness, and clinical care that have occurred recently in the field of pediatric AKI.

\section{The definition of AKI: speaking the same language}

The term acute kidney injury has replaced the dated "all or nothing" concept of acute renal failure $[1,2]$. The term "injury" highlights the spectrum of organ injury that may occur and the evolving nature of these events; clinicians are thus prompted to recognize and intervene early in the course of AKI rather than waiting until organ failure [3]. The operational definition of AKI has evolved from more than 35 divergent definitions to the modern definition. The development of standardized, multidimensional, staged AKI definitions has revolutionized our understanding of the epidemiology and impact of AKI on outcomes and our ability to interpret data across studies in a meaningful way. These systems define AKI according to stage of severity based on graded changes in serum creatinine (or estimated creatinine clearance) from a defined baseline and/or urine output. The evolution in the definition of AKI began in 2004 when the Acute Dialysis Quality Initiative proposed the RIFLE criteria, which included three stages of AKI: risk, injury, and failure and two outcomes: loss and end-stage kidney disease [4]. In 2007, a pediatric version of the RIFLE criteria, the pediatric RIFLE (pRIFLE) was developed and validated based on changes in estimated creatinine clearance [5]. Based upon the observations that even small $(0.3 \mathrm{mg} / \mathrm{dl})$ increases in creatinine were associated with increased in-hospital morbidity and mortality, the Acute Kidney Injury Network (AKIN) definition was proposed in 2007 [3]. In order to harmonize the subtle differences in these definitions, the Kidney Disease:

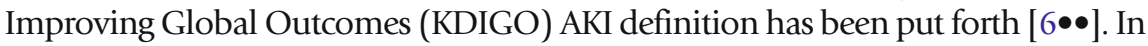
pediatric patients, the KDIGO AKI definition and staging should be utilized to

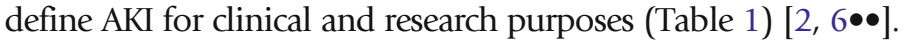




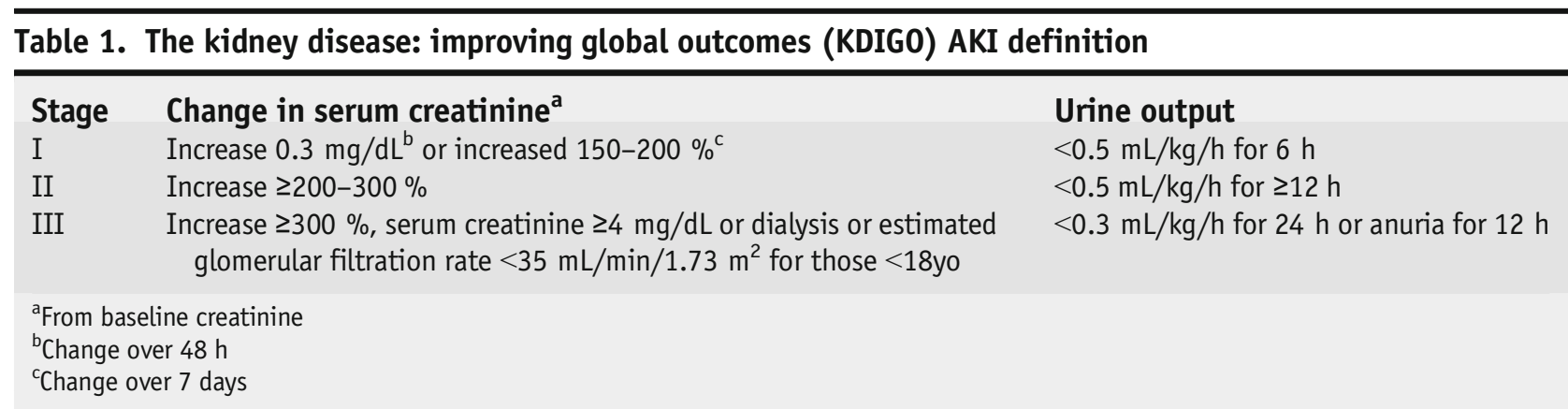

\section{Advances in clinical awareness: understanding the epidemiology and impact of AKI on outcomes}

The utilization of standardized definitions of AKI has allowed for the publication of updated epidemiologic studies highlighting the shift in underlying AKI etiologies. In developing countries, infection and primary renal diseases remain the most common causes of AKI [7-9]. In developed countries, while primary renal disease still accounts for a majority of AKI cases in otherwise healthy children, the etiology of AKI in hospitalized children is often multifactorial and reflective of comorbid conditions (e.g., bone marrow transplantation, need for extracorporeal membrane oxygenation) [10-14]. Most importantly, in each of these populations, AKI has been shown to be common and associated with adverse outcomes. We will review the incidence and outcomes associated with AKI in recent studies of some exemplar populations.

Among critically ill pediatric patient populations, the incidence of AKI is 10$25 \%[12-14]$ and can be as high as $82 \%$ in certain high risk populations, such as those that are mechanically ventilated [5]. These studies have shown an association between AKI and adverse outcomes including prolonged mechanical ventilation, longer intensive care unit (ICU) stays, and increased risk of mortality. For example, Alkandari et al. demonstrated an association between AKI and increased mortality (OR 3.7) that was independent of severity of illness in a cohort of 2106 patients; similar findings were reported by Selewski et al.

(OR 3.4) in a cohort of 3009 critically ill children [13, 14].

Children with congenital heart disease represent one of the most extensively studied pediatric populations at risk for AKI. The incidence in this population is high ranging from 30 to $52 \%$, with neonates representing the most vulnerable subpopulation with an incidence approaching $60 \%$ [15-18]. Post-cardiac surgery AKI is associated with increased risk of in-hospital mortality, duration of intensive care unit stay and overall hospitalization, time to extubation, and need for inotropic support. Several risk factors for the development of AKI in these patients have been identified including prolonged cardiopulmonary bypass time, complexity of surgical repair, degree of hypothermia, circulatory arrest, and postoperative low cardiac output syndromes [16, 18, 19].

Nephrotoxic medication exposure represents an important AKI risk factor, one that is potentially modifiable. Aminoglycosides represent one of the most potentially nephrotoxic medications in widespread use in hospitals: Zappitelli 
and colleagues reported a $20 \%$ incidence of AKI among children with $\geq 5$ days of aminoglycoside exposure [20]. Misurac et al. showed that non-steroidal antiinflammatory drugs (NSAID) induced AKI accounted for $2.7 \%$ of the episodes of AKI over an 11-year period even when used at recommended dosages. The presence of volume depletion at the time of NSAID use was a major risk factor for higher degree of injury. Patients $<5$ years of age were more likely to need dialysis, intensive care unit admission, and longer hospital stays. Cost estimates for the care of children with NSAID-associated AKI approached $\$ 400,000$ over an 11-year period [21]. Similarly, in a study of non-critically ill children, Moffett and Goldstein demonstrated that the use of three or more nephrotoxic medications had higher rates of AKI, longer hospital stays, and double the hospital costs ( $\$ 82,600$ vs. $\$ 48,300)$ [22].

Awareness of the significant impact of nephrotoxic medication exposure on the development of AKI, worse outcomes, and increased hospital costs has led to efforts to improve monitoring in these patients at the hospital level. Quality improvement initiatives aimed at nephrotoxic medication exposure screening and AKI detection using electronic medical record surveillance are ongoing and have shown early success.

Goldstein et al. reported one such system that utilized the electronic medical record to identify patients at risk of AKI (intravenous aminoglycosides $\geq 3$ days or $\geq 3$ nephrotoxic medications) in a near real-time manner using a multi-disciplinary approach including pharmacy support. The system then triggers more diligent renal function monitoring (daily creatinine measures). The authors demonstrated feasibility of the implementation of such a system at an institutional level; their efforts resulted in a $42 \%$ reduction in AKI intensity [23•].

\section{Advances in novel biomarkers: moving beyond serum creatinine and urine output}

Over the past decade, there have been extraordinary advances in the understanding of the diagnosis, epidemiology, and impact of AKI on outcomes in hospitalized patients. Despite these advances, there have not been any successful trials that have demonstrated effective treatment for or amelioration of AKI. In fact, current therapies are limited to supportive care including providing nutritional support, limiting nephrotoxic medications, insuring adequate renal perfusion, and managing sequelae of AKI (fluid overload, electrolyte abnormalities, uremia) [24•]. Failure to develop successful interventions in AKI is in large part related to the reliance on serum creatinine as our primary AKI biomarker. The limitations of serum creatinine have been well described and include a delay in rise of as much as $48 \mathrm{~h}$ after the injury and an inability to distinguish between functional changes and structural damage. These limitations have hampered the ability of clinicians and researchers to recognize AKI early enough to intervene in a meaningful way. As a result, there has been a significant amount of research to identify novel biomarkers of function and damage, measured in both urine and plasma, to discriminate more effectively the mechanism and timing of injury, especially when multiple biomarkers are used in combination [25]. 
The development of novel biomarkers to predict the development of AKI in a timely manner is essential to improving outcomes in children with AKI. It is important to distinguish these novel AKI biomarkers from functional biomarkers such as serum creatinine and cystatin C. Novel biomarkers classically include those that detect damage; some of these include urine neutrophil gelatinase-associated lipocalin (NGAL), kidney injury molecule-1 (KIM-1), interleukin 18 (IL-18), liver fatty acid binding protein, and others [26]. These have been extensively studied in children undergoing congenital heart surgery and cardiopulmonary bypass [27-29]. In these studies, the biomarkers rose between 2 and $12 \mathrm{~h}$ after cardiopulmonary bypass and successfully predicted $\mathrm{AKI}$, as defined by rise in serum creatinine, at $48 \mathrm{~h}$ following surgery [27]. These biomarkers are still undergoing rigorous testing and validation before they will be ready for widespread use in clinical care. In addition, while these biomarkers and others have successfully predicted AKI in at-risk populations, their predictive abilities have suffered when they are utilized non-selectively. As a result, there have been calls to improve pre-test probability by testing biomarkers only in "at risk" populations identified by clinical parameters (i.e., the concept of "renal angina") as is described below $[24 \bullet, 30]$.

\section{Advances in our understanding of pathophysiology and AKI diagnosis: moving beyond pre-renal, intrinsic renal, and post-renal}

A key to investigating the cause of AKI includes an understanding of the common etiologies in a particular patient population and a systematic, ordered approach to the diagnosis. A commonly utilized system for identifying AKI etiologies is to categorize potential insults as pre-renal, intrinsic, and post-renal. Even though this framework is easy to use, it is important to remember that this approach is an oversimplification of AKI pathophysiology. AKI is a clinically heterogeneous condition that involves multiple inflammatory, immunologic, cellular injury, adaptive, and autoregulatory pathways such that not all AKI events, even those within the same anatomic category, have the same underlying mechanism of injury [31]. The anatomic approach is thus imprecise in its ability to describe adequately the nature of the injury and hence the appropriate therapeutic intervention [32]. For instance, not all patients with "pre-renal" AKI-such as some patients with congestive heart failure or nephrotic syndrome-should receive aggressive volume resuscitation. While this remains an evolving area of research, a modern approach to AKI likely involves the incorporation of risk stratification, functional bedside tests, and a combination of functional and damage biomarkers.

As a result of the limitations of current biomarkers in terms of precision, timeliness, and prognostic ability, Basu et al. proposed the use of a collection of clinical risk factors and signs of kidney disease as a framework for identifying and stratifying patients most at risk for developing AKI. This concept emerged as "renal angina" and is analogous to the process of assessing the risk for myocardial infarction in a patient presenting with chest pain $[24 \bullet, 26]$. Clinical risk factors include those that are known to be associated with increased AKI risk 
(e.g., mechanical ventilation, bone marrow transplantation); these are combined with clinical and laboratory evidence of kidney injury (e.g., fluid overload and changes in serum creatinine) to provide a framework for stratifying patients into moderate-risk, high-risk, and very-high-risk categories. Thus, a patient who recently underwent bone marrow transplantation and is now intubated, receiving vasopressor medications and demonstrating $10 \%$ fluid overload would require smaller changes in serum creatinine or urine output to be considered at very high risk for AKI than would an otherwise healthy patient receiving nephrotoxic medications for an infection [30]. The renal angina construct is outlined in Tables 2 and 3 is calculated by multiplying risk * injury yielding a score of 1-40.

Basu et al. recently published the first study describing the derivation and validation of the renal angina index using four separate cohorts of critically ill

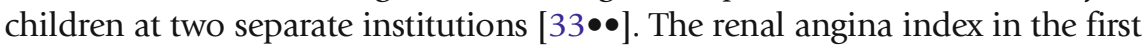
$8 \mathrm{~h}$ of PICU admission was able to predict stage 2 or 3 AKI successfully at $72 \mathrm{~h}$ of ICU admission. A renal angina index score of $\geq 8$ was defined as renal angina positive. The renal angina index performed better than severity of illness scores and KDIGO AKI stage at admission. Most importantly, patients who did not have signs of renal angina had a negative predictive value of $>92 \%$ for the development of severe AKI on day 3 . The authors suggest that the renal angina index can be utilized to identify patients who would benefit from further testing, such as with novel biomarkers. Utilizing such a schema, Basu et al. showed that in critically ill children with sepsis novel AKI biomarkers had poor discrimination when utilized alone, but when added to the renal angina index their ability to predict severe AKI improved significantly [34]. The Assessment of Worldwide Acute Kidney Injury, Renal Angina and Epidemiology in critically ill children (AWARE) study is a multicenter prospective observational cohort study that will seek to further validate this concept in critically ill children from over 32 institutions [35]. The purpose of this project is to develop a rich description of pediatric AKI epidemiology in the largest cohort of its kind as well as provide further validation of the renal angina index and its prognostic and diagnostic abilities.

Another avenue of active research prompted by the lack of timely biomarkers is the development of a bedside test of kidney function using diuretics. Chawla et al. have proposed the "furosemide stress test (FST)" as a simple, functional bedside test to help predict a patient's likelihood for progressing to severe AKI and consequent need for renal replacement therapy [36]. A one-time dose of furosemide, 1.5 or $1 \mathrm{mg} / \mathrm{kg}$ dose if furosemide naïve, is given, and urine output is monitored for the next $6 \mathrm{~h}$. The authors found that patients who were more likely to progress to AKI stage 3 had significantly less urine output following furosemide administration (urine volume $<200 \mathrm{mls}$ in adult

\section{Table 2. Renal angina index-risk}




\begin{tabular}{lll}
\hline Table 3. Renal angina index-injury & & Sercent fluid overload \\
\hline Decrease in creatinine clearance & $\leq 5$ & 1 \\
No change & $5-9.99$ & 2 \\
$0-24.99 \%$ & 10 to 14.99 & 4 \\
$25-49.99 \%$ & $\geq 15$ & 8 \\
$\geq 50 \%$ & &
\end{tabular}

patients) after $2 \mathrm{~h}$. A recent follow-up study showed this functional test may be as good if not better than individual biomarkers (e.g., urine NGAL, IL-18, KIM1 , and others) in its ability to predict progression to AKI stage 3 [37]. These studies were conducted in a sample of adult patients and would require further validation in pediatric studies. However, this maneuver could be applied easily to children with AKI. In practice, those who do not have a good urinary response to furosemide should be monitored more closely and have a lower threshold for initiating renal replacement therapy rather than waiting a little longer for renal recovery.

\section{Prevention and treatment of AKI}

As stated above, in lieu of definitive AKI treatments, supportive care remains the mainstay of treatment. Below, we will review several medications that have been studied as treatments and/or preventative measures for AKI, as well as key aspects of supportive care.

A number of therapies have been studied as potential interventions for AKI including diuretics, dopamine, fenoldopam, theophylline/aminophylline, and rasburicase. Furosemide is one of the most extensively utilized and studied of these agents. Furosemide may augment urine output in some patients, but has been not been shown to prevent the development of AKI or ameliorate its course $[6 \bullet \bullet, 38]$. Dopamine, an endogenous catecholamine, has been of interest in the prevention and treatment of AKI because of its vasodilatory effects on the renal vasculature via the dopamine- 1 and -2 receptors and its ability to enhance renal blood flow. Despite showing promise in animal models, dopamine has also not been shown to be of benefit in the prevention or treatment of AKI in several controlled trials [39]. Fenoldopam is a highly selective dopamine type 1 receptor agonist that preferentially dilates the renal and splanchnic vasculature. Fenoldopam has demonstrated some modest benefit in a small, single-center study of infants undergoing cardiopulmonary bypass for congenital heart disease repair and warrants further study [40].

Recently theophylline/aminophylline and rasburicase have shown promise in small single center studies as measures that may prevent the development of or progression to severe AKI in select patient populations. Theophylline and aminophylline act by inhibiting adenosine-induced vasoconstriction. Theophylline has been shown to prevent the development of AKI in asphyxiated newborns [41-43]. Theophylline has not been extensively studied in critically ill children with AKI. Aminophylline has shown promise in children who suffer AKI following cardiac surgery, where it was associated with improved renal excretory function and urine output [44]. A randomized double 
blinded trial of aminophylline in children undergoing cardiopulmonary bypass recently concluded. In addition, uric acid because of its multiple deleterious effects on renal function has received attention as a novel therapeutic target in AKI. Rasburicase is a recombinant urate oxidase that catalyzes the conversion of uric acid to allantoin. This drug has been utilized extensively to treat hyperuricemia and thus help to prevent AKI in the context of tumor lysis syndrome. Several recent case reports have shown successful use of rasburicase in the treatment of AKI associated with severe hyperuricemia in newborns and in pediatric patients with hemolytic uremic syndrome and rhabdomyolysis [4547]. Additional study is needed before firm recommendations regarding the use of these therapies can be made.

Until we have proven preventative or interventional treatments, care of children with AKI involves the employment of measures to prevent worsening kidney injury and minimize sequelae such as fluid overload and electrolyte abnormalities. A multidisciplinary approach is critical. For example, children with AKI have reduced drug clearance and are at higher risk for additional nephrotoxic medication-associated injury. Thus, daily evaluation of patient medication lists and dosages by a team of clinicians and pharmacists with expertise in AKI management should be utilized to optimize medication management. Similarly, children with AKI have changes in their electrolyte handling, especially potassium and phosphorus homeostasis, and in their volume requirements. These changes may vary from patient to patient depending on the mechanism of injury and level of residual urine output. Moreover, AKI represents a catabolic state, particularly in critically ill patients. Recent studies have shown that critically ill children with AKI are underfed with regard to protein and energy [48]. As a result, the comprehensive care of children with AKI, particularly the critically ill, should involve a dietician in order to optimize nutritional status. The inability to provide adequate nutrition or maintain metabolic control these are indications for more aggressive interventions including renal replacement therapy (RRT).

\section{Advances in the care of the patient with severe AKI: recognizing the impact of fluid overload and utilizing renal replacement therapy}

Over the past decade, RRT has transitioned from a therapy used as a "last-ditch effort" for patients in renal failure to a timely therapy directed at supporting the patient with kidney injury by preventing worsening outcomes and facilitating renal recovery. Depending on a center's expertise and resources, a variety of RRT modalities may be used including peritoneal dialysis (PD), intermittent hemodialysis (IHD), and continuous renal replacement therapy (CRRT) [49]. PD is often the modality of choice in smaller patients, especially critically ill neonates, as it is technically easier to implement without need for vascular access or large extracorporeal circuits. IHD is favored for use in children with intoxications or inborn errors in metabolism. Over the last 30 years, CRRT has become the modality of choice for providing RRT in critically ill children [50] because of its ability to provide slower and more controlled fluid removal that avoids the large fluid shifts that may occur with other modalities. 
The indications for RRT include uremia (typically BUN $>100 \mathrm{mg} /$ $\mathrm{dL}$ ), electrolyte abnormalities (particularly potassium and acidosis), inability to provide adequate nutrition, and fluid overload. Fluid overload currently represents one of the most common indications for initiation of RRT in critically ill children and warrants further discussion. The pediatric literature has been at the forefront of identifying the impact of fluid overload on mortality in critically ill children [51$53,54 \bullet \bullet, 55,56]$. These observational studies suggest that fluid overload 10-20\% represents a critical point for intervention with RRT. Using data from the Prospective Pediatric CRRT Registry, a multi-center study at 13 sites, Sutherland et al. showed that children who had $>20 \%$ fluid overload at the time of CRRT initiation had increased mortality (OR 8.5) [54••]. The deleterious effects of fluid overload have been demonstrated in other critically ill pediatric populations not receiving CRRT as well, including those requiring mechanical ventilation, in those following heart surgery, and those with shock [57•, 5860]. The American College of Critical Care Medicine now includes guidelines for addressing fluid balance in pediatric and neonatal patients with septic shock who amass $>10 \%$ volume overload during initial resuscitation [61]. RRT should be considered in patients who are fluid overloaded, especially if they have escalating ventilatory requirements, restricted nutrition, and need for large volumes of medications or blood products.

Wide acceptance of CRRT as an early therapy in critically ill children and neonates has been hampered by technical challenges of providing this therapy using equipment that was designed for adults but adapted for children. This is especially true for neonates and small infants. One of the most exciting new advances in the field of neonatal AKI has been the recent development of CRRT machines and systems designed specifically with the smallest of patients in mind. CRRT systems such as CARPEDIEM $^{\circledR}$ (Cardio-Renal Pediatric Dialysis Emergency Machine) and NIDUS ${ }^{\circledR}$ (Newcastle Infant Dialysis and Ultrafiltration System) have smaller extracorporeal volumes, lower required blood flows and ability to interface with smaller vascular catheters, making them ideal for use with small infants $[62,63]$. These machines are already in use outside the USA and show great promise; we are currently awaiting clinical trials for approval of these machines or use in the USA.

\section{Future directions: understanding the long-term implications of AKI and increasing awareness of the issue}

Previously, it was assumed that those who survive an episode of AKI would recover without long-term sequelae; however, follow-up data from children and adults who have suffered an AKI event suggests that survivors are at risk of developing chronic kidney disease (CKD) [64••, $65,66]$. Coca et al. recently showed that adults who experience an episode of AKI have an increased risk of developing CKD with a hazard ratio of 8.8 (95\% CI 3.1-25.5) [66]. 
Although the pediatric literature on the long-term effects of AKI is not as robust as that in adults, there is growing evidence that pediatric patients are also at risk of developing CKD following AKI events. In a prospective cohort study of children who experienced AKI in a tertiary center PICU, $10 \%$ of children developed CKD within 1 to 3 years, with an additional $50 \%$ of patients "at risk"

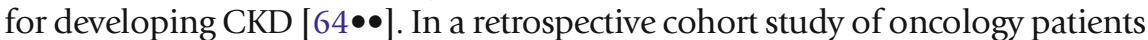
exposed to a nephrotoxic medication, $70 \%$ of patients who had an AKI event developed reduced eGFR, hyperfiltration, proteinuria, or hypertension within 6 months of that episode [67]. Finally, a recent meta-analysis evaluating the long-term outcomes of AKI in children has confirmed an increased risk of proteinuria, hypertension, and GFR $<90 \mathrm{~mL} / \mathrm{min} / 1.73 \mathrm{~m}^{2}$ following an episode of AKI [65]. From this data, it has become clear that children who have had an episode of AKI warrant follow-up.

The KDIGO guidelines include expert opinion about follow-up for adult patients after an episode of AKI. These patients should be seen by their primary physician within 3 months of the event. If signs of CKD (e.g., hypertension, proteinuria, elevated serum creatinine) are present, the referral to a specialist is necessary. While these recommendations are likely pertinent to children, currently there is not enough firm evidence to develop evidence-based practices. General pediatricians should consider children who have suffered AKI at increased risk and monitor blood pressure with consideration of further testing on a case by case basis.

To answer questions about appropriate long-term follow-up and many other issues pertaining to pediatric AKI, large longitudinal multi-center studies are needed. Pediatric AKI research has typically been hampered by small sample sizes and single-center studies that are often retrospective in design. The Assessment of Worldwide AKI, Renal Angina and Epidemiology (AWARE) study represents a major effort in this area. Initial data from this project are expected soon and will undoubtedly move the field of pediatric AKI much further into the future.

\section{Conclusion}

While the care of children who suffer AKI episodes is still hampered by the lack of a definitive "cure," there have been many major advances in the field of pediatric AKI, both in the laboratory and at the bedside. Standardized definitions have allowed for better identification of AKI in patients and comparison of data across studies. The development of more sensitive and specific AKI biomarkers is under way and will likely revolutionize our ability to intervene early enough in the course to develop and re-test previously proposed treatments and improve outcomes. The development of clinical frameworks like the renal angina score allows for earlier recognition of AKI at the bedside using a more robust combination of clinical signs and symptoms as well as functional and damage biomarkers. Our understanding of the impact of fluid overload is prompting earlier intervention and more effective utilization of renal replacement therapy. Above all, there is growing awareness of the scope of the problem and the impact of AKI on both short- and long-term outcomes. The development of a large, collaborative networks such as that for AWARE represents a major step forward in our ability to answer important questions in this field. 


\section{Compliance with Ethical Standards}

\section{Conflict of Interest}

Jennifer G. Jetton declares that she has no conflict of interest. Erika T. Rhone declares that she has no conflict of interest. Matthew W. Harer declares that he has no conflict of interest. Jennifer R. Charlton declares that she has no conflict of interest. David T. Selewski declares that he has no conflict of interest.

Human and Animal Rights and Informed Consent

This article does not contain any studies with human or animal subjects performed by any of the authors.

\section{References and Recommended Reading}

Papers of particular interest, published recently, have been

highlighted as:

- Of importance

-• Of major importance

1. Selewski DT, Symons JM. Acute kidney injury. Pediatrics in review/American Academy of Pediatrics. 2014;35:30-41.

2. Fortenberry JD, Paden ML, Goldstein SL. Acute kidney injury in children: an update on diagnosis and treatment. Pediatr Clin N Am. 2013;60:669-88.

3. Mehta RL, Kellum JA, Shah SV, Molitoris BA, Ronco C, Warnock DG, et al. Acute Kidney Injury Network: report of an initiative to improve outcomes in acute kidney injury. Crit Care. 2007;11:R31.

4. Bellomo R, Ronco C, Kellum JA, Mehta RL, Palevsky P. Acute renal failure-definition, outcome measures, animal models, fluid therapy and information technology needs: the Second International Consensus Conference of the Acute Dialysis Quality Initiative (ADQI) Group. Crit Care. 2004;8:R204-12.

5. Akcan-Arikan A, Zappitelli M, Loftis LL, Washburn KK, Jefferson LS, Goldstein SL. Modified RIFLE criteria in critically ill children with acute kidney injury. Kidney Int. 2007;71:1028-35.

6.• Kidney Disease: Improving Global Outcomes (KDIGO) Acute Kidney Injury Work Group. KDIGO Clinical Practice Guideline for Acute Kidney Injury. Kidney international Suppl. 2012: 1-138.

This manuscipt outlines consensus guidelines on the diagnosis and management of AKI. These guidelines were developed by international experts in the field of AKI from across medicine.

7. Esezobor CI, Ladapo TA, Lesi FE. Clinical profile and hospital outcome of children with severe acute kidney injury in a developing country. J Trop Pediatr. 2015;61:54-60.

8. Krishnamurthy S, Narayanan P, Prabha S, Mondal N, Mahadevan S, Biswal N, et al. Clinical profile of acute kidney injury in a pediatric intensive care unit from Southern India: a prospective observational study. Indian journal of critical care medicine : peer-reviewed, official publication of Indian Society of Critical Care Medicine. 2013;17:207-13.
9. Naik S, Sharma J, Yengkom R, Kalrao V, Mulay A. Acute kidney injury in critically ill children: risk factors and outcomes. Indian journal of critical care medicine : peer-reviewed, official publication of Indian Society of Critical Care Medicine. 2014;18:129-33.

10. Sutherland SM, Ji J, Sheikhi FH, Widen E, Tian L, Alexander SR, et al. AKI in hospitalized children: epidemiology and clinical associations in a national cohort. Clin J Am Soc Nephrol. 2013;8:1661-9.

11. Hui-Stickle S, Brewer ED, Goldstein SL. Pediatric ARF epidemiology at a tertiary care center from 1999 to 2001. Am J Kidney Dis. 2005;45:96-101.

12. Schneider J, Khemani R, Grushkin C, Bart R. Serum creatinine as stratified in the RIFLE score for acute kidney injury is associated with mortality and length of stay for children in the pediatric intensive care unit. Crit Care Med. 2010;38:933-9.

13. Alkandari O, Eddington KA, Hyder A, Gauvin F, Ducruet $\mathrm{T}$, Gottesman R, et al. Acute kidney injury is an independent risk factor for pediatric intensive care unit mortality, longer length of stay and prolonged mechanical ventilation in critically ill children: a two-center retrospective cohort study. Crit Care. 2011;15:R146.

14. Selewski DT, Cornell TT, Heung M, Troost JP, Ehrmann BJ, Lombel RM, et al. Validation of the KDIGO acute kidney injury criteria in a pediatric critical care population. Intensive Care Med. 2014;40:1481-8.

15. Blinder JJ, Goldstein SL, Lee VV, Baycroft A, Fraser CD, Nelson D, et al. Congenital heart surgery in infants: effects of acute kidney injury on outcomes. J Thorac Cardiovasc Surg. 2012;143:368-74.

16. Li S, Krawczeski CD, Zappitelli M, Devarajan P, Thiessen-Philbrook H, Coca SG, et al. Incidence, risk factors, and outcomes of acute kidney injury after pediatric cardiac surgery: a prospective multicenter study. Crit Care Med. 2011;39:1493-9.

17. Morgan CJ, Zappitelli M, Robertson CM, Alton GY, Sauve RS, Joffe AR, et al. Risk factors for and outcomes 
of acute kidney injury in neonates undergoing complex cardiac surgery. J Pediatr. 2013;162:120-7. e121.

18. Esch JJ, Salvin JM, Thiagarajan RR, Del Nido PJ, Rajagopal SK. Acute kidney injury after Fontan completion: risk factors and outcomes. J Thorac Cardiovasc Surg. 2015; 150:190-7.

19. Chiravuri SD, Riegger LQ, Christensen R, Butler RR, Malviya S, Tait AR, et al. Factors associated with acute kidney injury or failure in children undergoing cardiopulmonary bypass: a case-controlled study. Paediatr Anaesth. 2011;21:880-6.

20. Zappitelli M, Moffett BS, Hyder A, Goldstein SL. Acute kidney injury in non-critically ill children treated with aminoglycoside antibiotics in a tertiary healthcare centre: a retrospective cohort study. Nephrol Dial Transplant. 2011;26:144-50.

21. Misurac JM, Knoderer CA, Leiser JD, Nailescu C, Wilson AC, Andreoli SP. Nonsteroidal anti-inflammatory drugs are an important cause of acute kidney injury in children. J Pediatr. 2013;162:1153-9. 1159 e1151.

22. Moffett BS, Goldstein SL. Acute kidney injury and increasing nephrotoxic-medication exposure in noncritically-ill children. Clin J Am Soc Nephrol. 2011;6:856-63.

23. Goldstein SL, Kirkendall E, Nguyen H, Schaffzin JK, Bucuvalas J, Bracke T, et al. Electronic health record identification of nephrotoxin exposure and associated acute kidney injury. Pediatrics. 2013;132:e756-67.

This article describes the state of the art practice of utilizing the electronic medical record to identify nephrotoxic medication exposure associated AKI.

24.• Basu RK, Chawla LS, Wheeler DS, Goldstein SL. Renal angina: an emerging paradigm to identify children at risk for acute kidney injury. Pediatr Nephrol.

2012;27:1067-78.

This review article introduces and outlines the concept of Renal Angina.

25. Coca SG, Yalavarthy R, Concato J, Parikh CR. Biomarkers for the diagnosis and risk stratification of acute kidney injury: a systematic review. Kidney Int. 2008;73:1008-16.

26. Goldstein SL. Acute kidney injury biomarkers: renal angina and the need for a renal troponin I. BMC Med. 2011;9:135.

27. Krawczeski CD, Goldstein SL, Woo JG, Wang Y, Piyaphanee N, Ma Q, et al. Temporal relationship and predictive value of urinary acute kidney injury biomarkers after pediatric cardiopulmonary bypass. J Am Coll Cardiol. 2011;58:2301-9.

28. Alcaraz AJ, Gil-Ruiz MA, Castillo A, Lopez J, Romero C, Fernandez SN, et al. Postoperative neutrophil gelatinase-associated lipocalin predicts acute kidney injury after pediatric cardiac surgery*. Pediatr Crit Care Med. 2014;15:121-30.

29. Parikh CR, Devarajan P, Zappitelli M, Sint K, ThiessenPhilbrook H, Li S, et al. Postoperative biomarkers predict acute kidney injury and poor outcomes after pediatric cardiac surgery. J Am Soc Nephrol. 2011;22:1737-47.
30. Goldstein SL, Chawla LS. Renal angina. Clin J Am Soc Nephrol. 2010;5:943-9.

31. Symons JM. Moving beyond supportive care-current status of specific therapies in pediatric acute kidney injury. Pediatr Nephrol. 2014;29:173-81.

32. Endre ZH, Kellum JA, Di Somma S, Doi K, Goldstein SL, Koyner JL, et al. Differential diagnosis of AKI in clinical practice by functional and damage biomarkers: workgroup statements from the tenth Acute Dialysis Quality Initiative Consensus Conference. Contrib Nephrol. 2013;182:30-44.

33.• Basu RK, Zappitelli M, Brunner L, Wang Y, Wong HR, Chawla LS, et al. Derivation and validation of the renal angina index to improve the prediction of acute kidney injury in critically ill children. Kidney Int. 2014;85:659-67.

This is the sentinel study that was utilized to derive and validate the Renal Angina Index in criticallyill children.

34. Basu RK, Wang Y, Wong HR, Chawla LS, Wheeler DS, Goldstein SL. Incorporation of Biomarkers with the Renal Angina Index for Prediction of Severe AKI in Critically Ill Children. Clin J Am Soc Nephro. 2014;9:654-62.

35. Basu RK, Kaddourah A, Terrell T, Mottes T, Arnold P, Jacobs J, et al. Assessment of Worldwide Acute Kidney Injury, Renal Angina and Epidemiology in critically ill children (AWARE): study protocol for a prospective observational study. BMC Nephrol. 2015;16:24.

36. Chawla LS, Davison DL, Brasha-Mitchell E, Koyner JL, Arthur JM, Shaw AD, et al. Development and standardization of a furosemide stress test to predict the severity of acute kidney injury. Crit Care. 2013; 17:R207.

37. Koyner JL, Davison DL, Brasha-Mitchell E, Chalikonda DM, Arthur JM, Shaw AD, et al. Furosemide stress test and biomarkers for the prediction of AKI severity. J Am Soc Nephrol. 2015;26:2023-31.

38. Ho KM, Sheridan D. Meta-analysis of frusemide to prevent or treat acute renal failure. Brit Med J. 2006;333:420-3.

39. Friedrich JO, Adhikari N, Herridge MS, Beyene J. Metaanalysis: low-dose dopamine increases urine output but does not prevent renal dysfunction or death. Ann Intern Med. 2005;142:510-24.

40. Ricci Z, Luciano R, Favia I, Garisto C, Muraca M, Morelli S, Di Chiara L, Cogo P, Picardo S, (2011) Highdose fenoldopam reduces postoperative neutrophil gelatinase-associated lipocaline and cystatin $\mathrm{C}$ levels in pediatric cardiac surgery. Critical Care 15

41. Jenik AG, Cernadas JMC, Gorenstein A, Ramirez JA, Vain N, Armadans $\mathrm{M}$, et al. A randomized, doubleblind, placebo-controlled trial of the effects of prophylactic theophylline on renal function in term neonates with perinatal asphyxia. Pediatrics. 2000;105:no.-e45.

42. Bakr AF. Prophylactic theophylline to prevent renal dysfunction in newborns exposed to perinatal asphyxia - a study in a developing country. Pediatr Nephrol. 2005;20:1249-52.

43. Eslami Z, Shajari A, Kheirandish M, Heidary A. Theophylline for prevention of kidney dysfunction in 
neonates with severe asphyxia. Iran J Kidney Dis. 2009;3:222-6.

44. Axelrod DM, Anglemyer AT, Sherman-Levine SF, Zhu A, Grimm PC, Roth SJ, et al. Initial experience using aminophylline to improve renal dysfunction in the pediatric cardiovascular ICU. Pediatr Crit Care Med. 2014;15:21-7.

45. Acosta AA, Hogg RJ. Rasburicase for hyperuricemia in hemolytic uremic syndrome. Pediatr Nephrol. 2012;27:325-9.

46. Hobbs DJ, Steinke JM, Chung JY, Barletta GM, Bunchman TE. Rasburicase improves hyperuricemia in infants with acute kidney injury. Pediatr Nephrol. 2010;25:305-9.

47. Lin PY, Lin CC, Liu HC, Lee MD, Lee HC, Ho CS, et al. Rasburicase improves hyperuricemia in patients with acute kidney injury secondary to rhabdomyolysis caused by ecstasy intoxication and exertional heat stroke. Pediatr Crit Care Me. 2011;12:E424-7.

48. Kyle UG, Akcan-Arikan A, Orellana RA, Coss-Bu JA. Nutrition support among critically ill children with AKI. Clin J Am Soc Nephro. 2013;8:568-74.

49. Walters S, Porter C, Brophy PD. Dialysis and pediatric acute kidney injury: choice of renal support modality. Pediatr Nephrol. 2009;24:37-48.

50. Warady BA, Bunchman T. Dialysis therapy for children with acute renal failure: survey results. Pediatr Nephrol. 2000;15:11-3.

51. Goldstein SL, Currier H, Graf C, Cosio CC, Brewer ED, Sachdeva R. Outcome in children receiving continuous venovenous hemofiltration. Pediatrics.

2001;107:1309-12.

52. Hayes LW, Oster RA, Tofil NM, Tolwani AJ. Outcomes of critically ill children requiring continuous renal replacement therapy. J Crit Care. 2009;24:394-400.

53. Selewski DT, Cornell TT, Lombel RM, Blatt NB, Han YY, Mottes T, et al. Weight-based determination of fluid overload status and mortality in pediatric intensive care unit patients requiring continuous renal replacement therapy. Intensive Care Med. 2011;37:1166-73.

54.• Sutherland SM, Zappitelli M, Alexander SR, Chua AN, Brophy PD, Bunchman TE, et al. Fluid overload and mortality in children receiving continuous renal replacement therapy: the prospective pediatric continuous renal replacement therapy registry. Am J Kidney Dis. 2010;55:316-25.

This article describes the association of the degree of fluid overload at continuous renal replacement therapy initiation with mortality. The article represents the largest and most comprehensive publication on the impact of fluid overload inthis population to date.

55. Foland JA, Fortenberry JD, Warshaw BL, Pettignano R, Merritt RK, Heard ML, et al. Fluid overload before continuous hemofiltration and survival in critically ill children: a retrospective analysis. Crit Care Med. 2004;32:1771-6.

56. Gillespie RS, Seidel K, Symons JM. Effect of fluid overload and dose of replacement fluid on survival in hemofiltration. Pediatr Nephrol. 2004;19:1394-9.
57. Arikan AA, Zappitelli M, Goldstein SL, Naipaul A, Jefferson LS, Loftis LL. Fluid overload is associated with impaired oxygenation and morbidity in critically ill children. Pediatr Crit Care Med. 2012;13:253-8.

This article describes the implications of fluid overload in a critically ill patient population that is not on continuous renal replacement therapy. In this population of mechanically ventilated patients fluid overload is associated with impaired oxygenation and outcomes.

58. Seguin J, Albright B, Vertullo L, Lai P, Dancea A, Bernier $\mathrm{PL}$, et al. Extent, risk factors, and outcome of fluid overload after pediatric heart surgery*. Crit Care Med. 2014;42:2591-9.

59. Hassinger AB, Wald EL, Goodman DM. Early postoperative fluid overload precedes acute kidney injury and is associated with higher morbidity in pediatric cardiac surgery patients. Pediatr Crit Care Med. 2014;15:131-8.

60. Bhaskar P, Dhar AV, Thompson M, Quigley R, Modem V. Early fluid accumulation in children with shock and ICU mortality: a matched case-control study. Intensive Care Med. 2015;41:1445-53.

61. Brierley J, Carcillo JA, Choong K, Cornell T, Decaen A, Deymann A, et al. Clinical practice parameters for hemodynamic support of pediatric and neonatal septic shock: 2007 update from the American College of Critical Care Medicine. Crit Care Med. 2009;37:666-88.

62. Coulthard MG, Crosier J, Griffiths C, Smith J, Drinnan $\mathrm{M}$, Whitaker $\mathrm{M}$, et al. Haemodialysing babies weighing $<8 \mathrm{~kg}$ with the Newcastle infant dialysis and ultrafiltration system (Nidus): comparison with peritoneal and conventional haemodialysis. Pediatr Nephrol. 2014;29:1873-81.

63. Ronco C, Garzotto F, Brendolan A, Zanella M, Bellettato M, Vedovato S, et al. Continuous renal replacement therapy in neonates and small infants: development and first-in-human use of a miniaturised machine (CARPEDIEM). Lancet. 2014;383:1807-13.

64.• Mammen C, Al Abbas A, Skippen P, Nadel H, Levine D, Collet JP, et al. Long-term risk of CKD in children surviving episodes of acute kidney injury in the intensive care unit: a prospective cohort study. Am J Kidney Dis. 2012;59:523-30.

This article systematically describes for the first time the implications of an episode of AKI on the development of chronic kidney disease. This long-term follow-up study demonstrated that critically ill children who suffer an episode of AKI are at risk for progression to chronic kidney disease.

65. Greenberg JH, Coca S, Parikh CR. Long-term risk of chronic kidney disease and mortality in children after acute kidney injury: a systematic review. BMC Nephrol. 2014;15.

66. Coca SG, Singanamala S, Parikh CR. Chronic kidney disease after acute kidney injury: a systematic review and meta-analysis. Kidney Int. 2012;81:442-8.

67. Menon S, Kirkendall ES, Nguyen H, Goldstein SL. Acute kidney injury associated with high nephrotoxic medication exposure leads to chronic kidney disease after 6 months. J Pediatr-Us. 2014;165:522. 